OPEN ACCESS

Edited by:

Yuji Morita,

Aichi Gakuin University, Japan

Reviewed by:

Antoine Danchin,

CHU Pitié-Salpêtrière, France

Suresh Korpole,

Institute of Microbial Technology, India

*Correspondence:

S. Adline Princy

adlineprinzy@biotech.sastra.edu

Specialty section:

This article was submitted to

Antimicrobials, Resistance and

Chemotherapy,

a section of the journal

Frontiers in Microbiology

Received: 13 January 2016 Accepted: 23 May 2016

Published: 06 June 2016

Citation:

Kaur G, Balamurugan P, Uma Maheswari C, Anitha A and Princy SA (2016) Combinatorial Effects of Aromatic 1,3-Disubstituted Ureas and

Fluoride on In vitro Inhibition of

Streptococcus mutans Biofilm

Formation. Front. Microbiol. 7:861

doi: 10.3389/fmicb.2016.00861

\section{Combinatorial Effects of Aromatic 1,3-Disubstituted Ureas and Fluoride on In vitro Inhibition of Streptococcus mutans Biofilm Formation}

\author{
Gurmeet Kaur $^{1}$, P. Balamurugan ${ }^{1}$, C. Uma Maheswari ${ }^{2}$, A. Anitha ${ }^{2}$ and S. Adline Princy ${ }^{1 *}$ \\ ${ }^{1}$ Quorum Sensing Laboratory, Centre for Research in Infectious Diseases, School of Chemical and Biotechnology, SASTRA \\ University, Thanjavur, India, ${ }^{2}$ Organic Synthesis Group, Department of Chemistry, School of Chemical and Biotechnology, \\ SASTRA University, Thanjavur, India
}

Dental caries occur as a result of disequilibrium between acid producing pathogenic bacteria and alkali generating commensal bacteria within a dental biofilm (dental plaque). Streptococcus mutans has been reported as a primary cariogenic pathogen associated with dental caries. Emergence of multidrug resistant as well as fluoride resistant strains of $S$. mutans due to over use of various antibiotics are a rising problem and prompted the researchers worldwide to search for alternative therapies. In this perspective, the present study was aimed to screen selective inhibitors against ComA, a bacteriocin associated $\mathrm{ABC}$ transporter, involved in the quorum sensing of $S$. mutans. In light of our present in silico findings, 1,3-disubstituted urea derivatives which had better affinity to ComA were chemically synthesized in the present study for in vitro evaluation of $S$. mutans biofilm inhibition. The results revealed that 1,3-disubstituted urea derivatives showed good biofilm inhibition. In addition, synthesized compounds exhibited potent synergy with a very low concentration of fluoride (31.25-62.5 ppm) in inhibiting the biofilm formation of $S$. mutans without affecting the bacterial growth. Further, the results were supported by confocal laser scanning microscopy. On the whole, from our experimental results we conclude that the combinatorial application of fluoride and disubstituted ureas has a potential synergistic effect which has a promising approach in combating multidrug resistant and fluoride resistant $S$. mutans in dental caries management.

Keywords: Streptococcus mutans, dental caries, fluoride resistant, quorum sensing, synergism, multidrug resistant

\section{INTRODUCTION}

Streptococcus mutans has been reported as a primary cariogenic bacterial pathogen in causing dental caries, along with other acidogenic bacteria such as Lactobacillus casei, Actinomyces (Kaur et al., 2015). In addition, this bacterium has also been reported to cause chronic infective endocarditis which may cause significant morbidity and mortality (Persson, 2012). S. mutans has established various characteristic mechanisms for its unhindered growth and survival in the complex microbial community of the oral cavity. Major virulence factors such as acidogenicity, aciduricity, and the ability to synthesize extracellular polysaccharides from carbohydrates such as sucrose using enzymes such as glucosyltransferases (GTFs) and collagenanses, reported to have 
ability to bind and degrade collagen type I, a major component of dentin (Han et al., 2006) are a few among such mechanisms (Hasan et al., 2012). The glucan synthesis plays a crucial role in biofilm formation of dentine commonly known as dental plaque (Lynch et al., 2013). Recent advances in understanding the communication between bacterial communities have established that like various other bacteria, $S$. mutans also regulate the process of biofilm formation through quorum sensing (QS) system (Miller and Bassler, 2001). QS circuit in S. mutans consists of two component signal transduction system (TCSTS) that specifically detect and respond to the signaling peptide known as Competence Stimulating Peptide (CSP) (Kleerebezem et al., 1997). The CSP is synthesized as a propeptide by $\operatorname{com} C$ which is then processed and matured by an ABC transporter ComA with the help of an accessory protein ComB and finally secreted to the extracellular environment. The secreted peptide is detected by the ComD, a histidine kinase protein receptor resulting in phosphorylation of its cytoplasmic response regulator, ComR thus, enabling the cell to respond to the peptide via regulation of gene expression controlling various virulence factors such as genetic competence and biofilm formation (Kotake et al., 2008).

Biofilm formation is one of the significant characteristics in various infectious diseases as it provides various advantages to bacteria such as protection from host defense system, sequestration of nutrients, utilization of community benefits, and protection from various antimicrobials since it acts as a diffusion barrier for those agents to penetrate deep into the biofilms (Senadheera and Cvitkovitch, 2008). Targeting one of the crucial components involved in QS circuit can lead to biofilm inhibition (Qi et al., 2005; Rasmussen and Givskov, 2006; Ravichandiran et al., 2013). Development of novel drugs against biofilm formation, a major virulence factor in dental caries aids in the effective clearance of $S$. mutans when used in combination with very low concentrations of antimicrobials thus combating multidrug resistance (Chen et al., 2016).

Fluoride has been used long as an effective anti-caries agent in various commercial formulations and is the mainstay for caries prevention (Zheng et al., 2015). It exerts major effects by reducing enamel demineralization and enhancing remineralization of early caries lesions (Randall et al., 2014). However, fluoride does not provide complete protection in its currently used preparations and moreover, emergence of fluoride resistant $S$. mutans has also doubted the prolonged use of fluoride (Mitsuhata et al., 2014). Although, fluoride at higher concentrations helps in reduction of dental plaque and also inhibits the growth of dental pathogens, prolonged use of high concentrations of fluoride has caused various side effects such as fluorosis and weakened bones (Cavalli et al., 2011; Santos et al., 2013). Thus, if an additional agent could be administered synergistically with fluoride, it may lead to improved cariostatic and disruptive effects with respect to biofilm formation without increasing fluoride's exposure.

In view of this hypothesis, ComA was identified as a key molecule crucial for the initiation of QS system of Streptococcus, which belongs to a family of bacteriocin-associated ATPbinding cassette $(\mathrm{ABC})$ transporters. $\mathrm{ABC}$ transporters usually consists of three domains, i.e., N-terminal domain possessing the proteolytic peptidase activity, a transmembrane domain involving six membrane-spanning segments, and a C-terminal ATP-binding domain positioned toward the cytoplasmic face of the membrane. Proteolytic activity of peptidase domains of this family is believed to be involved in cleaving their cognate propeptides after the consensus Gly-Gly motif. The presence of a cysteine residue is essential to the sequence for the proteolytic activity of this family of ABC transporters (Kotake et al., 2008). Recently, the crystal structure of peptidase (PEP) domain involved in the QS pathway of Streptococcus was reported by Ishii et al. (2010) and its proteolytic activity substrate recognition mechanism was reported by Kotake et al. (2008). The key involvement of ComA in maturation and secretion of CSP made it as a favorable target for QS inhibition of S. mutans. Thus, the aims of the present study were (1) to screen selective inhibitors against ComA, using computational tools utilizing small compound library and synthesize the screened ligand along with its derivatives, (2) to evaluate the synthesized compounds for biofilm inhibition potential against $S$. mutans, and (3) to determine the combinatorial biological activity of the synthesized compounds at lower concentrations of fluoride.

\section{MATERIALS AND METHODS}

\section{Ligand and Protein Preparation}

The energy minimized 3D coordinates were generated for all ligands and ligand file was prepared for docking using Schrödinger LigPrep software (Xiao et al., 2011). An independent ligand dataset library Zinc database was used for this study (Irwin and Shoichet, 2005). Since none of the inhibitors have been reported for PEP-ComA, thus, comparison studies were not conducted with known inhibitors. The X-ray crystal structure of the PEP domain of ComA (PDB ID-3K8U) was retrieved from $\mathrm{PDB}$ database and processed for addition of polar hydrogen and Kollmann charges using Protein Preparation Wizard (PrepWizard) in Maestro (Schrödinger Suite) (Bernstein et al., 1978). The prepared protein was treated to be completely rigid for all docking procedures to minimize the excessive computation cost. A grid box encompassing the complete macromolecule was constructed and used for all docking runs in this study (Osguthorpe et al., 2012). The hydrogen bonds were optimized and protein minimization was carried out using the standard protein preparation protocol. The possible options available for protein minimization included hydrogen only or allatom with a criterion based on the root-mean-square deviation (RMSD) of the heavy atoms relative to their initial location. Water molecules were retained through the $\mathrm{H}$-bond optimization and minimization stages, as without water molecules, the protein could collapse, or the H-bond networks required for ligand binding would be disrupted. Although, prior to docking, all the water molecules (>5 $\AA$ ) were removed. The resultant protein structure was used for further docking studies with the prepared ligands.

\section{Molecular Docking Studies}

All docking calculations between macromolecule and ligand datasets were performed with Glide (Grid based LIgandDocking with Energetics) program in Schrödinger suite (Friesner et al., 
2004). All the datasets were executed in three consecutive steps. Precisely, HTVS mode (High-Throughput Virtual Screening), SP mode (Standard Precision), followed by XP mode (eXtraPrecision mode) using the default settings. All the prepared ligands were docked with the target protein using the induced fit docking (IFD) protocol (Friesner et al., 2006). Extensive sampling was conducted by SP mode which defines the interaction sites of ligand with the protein molecule. Resultant G-scores were considered as the ranking criteria for the selection of best docked ligands to the target protein.

\section{De novo Drug Designing and Lead Optimization}

The selected ligands were screened based on their respective glide scores, interaction pattern at the site of interest in the protein and the functional group of the compounds. The selected ligands were further run for clustering using Schrödinger Canvas module (Hartenfeller and Schneider, 2011). The 3D-Pharmacophore and binary fingerprinting studies optimized the ligands by ruling out the ligands with similar structures and their interaction with the protein. Clustering of ligands was followed by optimization of lead molecules with Schrödinger QuikProp for toxicity analysis of the selected ligands (Singh and Konwar, 2012). The parameters in ADMET and TOPKAT (TOxicityPrediction by Komputer Assisted Technology) was used for the final validation of the selected compounds and deemed to be the potential leads.

\section{Synthesis of Aromatic 1,3-Disubstituted Ureas}

The 1,3-disubstituted ureas were synthesized by a simple one pot reaction of aryl isocyanates with the selective amines (Mustafa et al., 2014). The synthesis process is shown as a reaction scheme in Figure 1A. For the synthesis of 1,3-disubstituted ureas, a solution of substituted aniline $(3.18 \mathrm{mmol})$ was mixed in 1,4dioxane $(5 \mathrm{~mL})$ and aryl isocyanate $(1.60 \mathrm{mmol})$ was added drop-wise to the reaction mixture and kept for stirring at room temperature for $18 \mathrm{~h}$. The progress of the reaction was monitored by thin layer chromatography. The difference in the $R_{\mathrm{f}}$ values of the reactants and products in the reaction mixture indicated the completion of the reaction (Figure 1B). After completion of the reaction, the reaction mixture was cooled down by addition of crushed ice which resulted in formation of products as solids. The solid, thus obtained was filtered and washed several times with cold distilled water followed by washing with hexane to obtain the pure product. The structures of the synthesized products were confirmed by ${ }^{1} \mathrm{H}$ and ${ }^{13} \mathrm{C}$ NMR spectroscopy.

\section{Bacterial Strains and Culture Conditions}

S. mutans MTCC 497 was received from Microbial Type Culture Collection (MTCC), Chandigarh, India and was used as a standard strain in this study. A wild type strain (WT) NG8 was kindly donated by Prof. L. Jeannine Brady, University of Florida, USA. Two clinical isolates of S. mutans 4SM (multidrug resistant) and 5SM (fluoride resistant) were received from JSS Medical College, Mysore, India. All the strains were cultured in brain heart infusion broth (BHIB)/agar (HiMedia) at $37^{\circ} \mathrm{C}$ under stationary condition. When needed, medium was supplemented with $2 \%$ sucrose. Sodium fluoride and Chlorhexidine diacetate were purchased from HiMedia (Mumbai, India.)

\section{Effect of 1, 3-Disubstituted Ureas on Biofilm Development in Static Biofilm Model}

Ninety six well polystyrene microtiter plates were used for determining minimum biofilm inhibitory concentration (MBIC) and minimum biofilm eradication concentration (MBEC) assay (Kolodkin-Gal et al., 2012; Wang et al., 2015). The assays were carried out against clinical as well as standard isolates of $S$. mutans using two-fold microdilution broth procedure as per the Clinical and Laboratory Standards Institute $\left(\mathrm{CLSI}^{1}\right)$ standards with slight modifications as described below (CLSI Approved Standard-Tenth Edition). S. mutans strains were grown overnight in BHIB. The overnight cultures were diluted (1:100) and the initial optical density of the bacterial cultures were adjusted to $0.08\left(\mathrm{OD}_{600}\right)$ to achieve $10^{8} \mathrm{CFU} / \mathrm{ml}$ approximately. The inoculum was inoculated in fresh medium along with varying concentrations of synthesized compounds ranging from 0.23 to $15 \mu \mathrm{M}$ as independent experiments (in triplicates). Plates were incubated for $24 \mathrm{~h}$ at $37^{\circ} \mathrm{C}$ without shaking. After incubation, the wells were washed twice with $200 \mu \mathrm{L}$ of phosphate buffered saline (PBS) gently to remove the nonadherent cells. Adherent cells in the biofilm were fixed by adding $200 \mu \mathrm{L}$ of $100 \%$ methanol prior to staining with $200 \mu \mathrm{L}$ of $0.2 \%(\mathrm{w} / \mathrm{v})$ crystal violet $(\mathrm{CV})$ for $20 \mathrm{~min}$. The excess stain was washed twice with PBS and the plates were air dried. The bound CV in the air dried plates were eluted with $200 \mu \mathrm{L}$ of $33 \%$ acetic acid. The biofilm was quantitatively determined by measuring the absorbance at $\mathrm{OD}_{595} \mathrm{~nm}$ in a microtiter plate reader (iMark, BIORAD, Japan). The lowest concentration in which the formation of biofilm is inhibited when compared to the untreated culture control is defined as MBIC i.e., $\geq 90 \%$ (for $\mathrm{MBIC}_{90}$ or $\mathrm{IC}_{90}$ ) or $\geq 50 \%$ (for $\mathrm{MBIC}_{50}$ or $\mathrm{IC}_{50}$ ).

Similarly, MBEC assay was performed as described elsewhere (Jennings et al., 2014). Briefly, the biofilm of the respective strains were allowed to grow in polystyrene 96 well microtiter plates for $18 \mathrm{~h}$. After incubation, the wells were washed twice with PBS to remove planktonic cells followed by addition of fresh medium along with varying concentrations of synthesized compounds. The plates were incubated for $3 \mathrm{~h}$ without shaking followed by CV staining of wells as described earlier. The absorbance at $\mathrm{OD}_{595}$ $\mathrm{nm}$ was measured. The lowest concentration in which the biofilm was eradicated when compared to the untreated culture control is defined as MBEC i.e., $\geq 90 \%$ (for $\mathrm{MBEC}_{90}$ or $\mathrm{EC}_{90}$ ) or $\geq 50 \%$ (for $\mathrm{MBEC}_{50}$ or $\left.\mathrm{EC}_{50}\right)$. All the assays were performed in triplicates.

\section{Checkerboard Microdilution Assay for Synergistic Studies}

Combinatorial effects between synthesized compounds and fluoride as well as chlorhexidine was evaluated by a two

\footnotetext{
${ }^{1}$ Clinical and Laboratory Standards Institute (CLSI). CLSI Approved StandardTenth Edition). Methods for Dilution Antimicrobial Susceptibility Tests for Bacteria That Grow AerobicallyCLSI document M07-A10 ISBN 1-56238-987-4; ISBN 1-56238-988-2.
} 

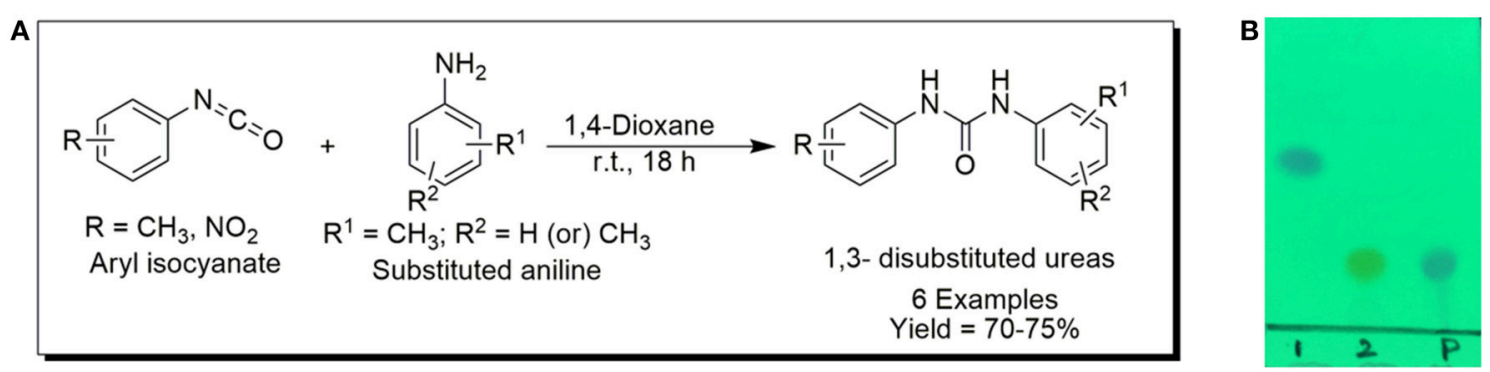

FIGURE 1 | (A) Synthesis scheme of 1,3-disubstituted ureas. (B) A thin layer chromatogram showing completion of the reaction (30\% Ethyl acetate:Hexane). Spot 1 represents reactant 1 (2,4,6-Trimethyl aniline), spot 2 represents (4-nitrophenyl isocyanate), and $\mathrm{P}$ represents their corresponding product

(1-mesityl-3-(4-nitrophenyl)urea). The difference in the $R_{f}$ values and color change of reactants to products indicates the completion of reaction.

dimensional microdilution assay using 96 well microtiter plate (Zheng et al., 2015). The final concentrations of fluoride ranged from 3.90 to $250 \mathrm{ppm}$ and that of chlorhexidine ranged from 0.003125 to $0.2 \%$. The concentration of fluoride was selected where it was not inhibiting bacterial growth and also was not acting as biofilm inhibiting agent. For chlorhexidine, $0.2 \%$ is the highest concentrations which is used in commercial formulations. For each combination, each synthesized compound was placed in horizontal rows while fluoride and chlorhexidine were placed into the vertical columns. Data were collected using microtiter plate reader (iMark, BIORAD, Japan) as turbidometric measurements of absorbance $\left(\mathrm{OD}_{595}\right)$ after incubation for $24 \mathrm{~h}$. The synergistic activity was assessed by the currently prevailing two most widely used approaches i.e., using fractional inhibitory concentration index (FICI) and Bliss independence model $(\Delta \mathrm{E}$ model).

\section{Synergy Interpretation Using $\mathrm{FICl}$}

To determine the effect of the combinatorial treatment to be synergistic, indifferent or antagonistic, FICI (a nonparametric method) was calculated (Subramaniam et al., 2014). The following formulas were used to calculate the FICI of a combination.

$$
\text { FICI }=\text { FIC of compound A }+ \text { FIC of compound B }
$$

Where,

FIC of compound $\mathrm{A}=$ (MIC of compound $\mathrm{A}$ in combination)/(MIC of compound A alone).

FIC of compound $\mathrm{B}=$ (MIC of compound $\mathrm{B}$ in combination)/(MIC of compound B alone).

Synergy was defined as an FIC index value of $\leq 0.5$. Indifference or absence of interaction was defined as FICI value $>0.5$ but $<4$. Antagonism was defined as an FICI of $>4$.

\section{Synergy Interpretation Using Bliss Independence Model}

The bliss independence model or BI theory (Goldoni and Johansson, 2007; Sun et al., 2008) is described by the following equation.

$$
\mathrm{I}_{\mathrm{i}}=\left(\mathrm{I}_{\mathrm{A}}+\mathrm{I}_{\mathrm{B}}\right)-\left(\mathrm{I}_{\mathrm{A}} \mathrm{X} \mathrm{I}_{\mathrm{B}}\right)
$$

Where,

$\mathrm{Ii}=$ predicted percentage of inhibition of the theoretical combination of drug $\mathrm{A}$ and $\mathrm{B}$.

$\mathrm{I}_{\mathrm{A}}=$ experimental percentages of inhibition of drug A.

$\mathrm{I}_{\mathrm{B}}=$ experimental percentages of inhibition of drug $\mathrm{B}$.

$$
\mathrm{I}=1-\mathrm{E}
$$

Where,

$\mathrm{E}=$ percentage of growth.

Substituting Equation (2) into (1), a resultant equation is given as

$$
\mathrm{E}_{\mathrm{i}}=\mathrm{E}_{\mathrm{A}} \mathrm{X \textrm {E } _ { \mathrm { B } }}
$$

Where,

$\mathrm{E}_{\mathrm{i}}=$ predicted growth percentage of the theoretical combination of drug $\mathrm{A}$ and $\mathrm{B}$.

$\mathrm{E}_{\mathrm{A}}=$ observed percentage of growth of drug $\mathrm{A}$.

$\mathrm{E}_{\mathrm{B}}=$ observed percentage of growth of drug $\mathrm{B}$.

Interaction is described by the difference $(\Delta E)$ between the predicted and the observed growth percentages at various concentrations as described by the following equation:

$$
\Delta \mathrm{E}=\mathrm{E}_{\text {predicted }}-\mathrm{E}_{\text {observed }}
$$

By the nonparametric approach described by Prichard et al. $\mathrm{E}_{\mathrm{A}}$ and $\mathrm{E}_{\mathrm{B}}$ were obtained directly from the experimental data. Due to the nature of interaction, testing with microtiter plates, and a two-fold dilution of either drug results in a $\Delta \mathrm{E}$ for each drug combination. In each of the three independent experiments, the observed growth percentages derived from the experimental data (observed values) were subtracted from the predicted percentages, and then the average difference of three experiments was calculated. When the average difference as well as its $95 \%$ confidence interval among the three replicates was positive, statistically significant synergy was claimed; when the difference as well as its $95 \%$ confidence interval was negative, significant antagonism was claimed. 


\section{Confocal Laser Scanning Microscopy Imaging of Biofilms}

Confocal imaging was performed on bacterial cells grown in the form of biofilms on the surface of glass slides. The glass slides were placed inside sterile $50 \mathrm{ml}$ centrifuge tubes containing BHIB with or without the compounds at concentrations that showed maximum biofilm inhibition activity in 96 well microtiter plate experiments (Auty et al., 2001). S. mutans was inoculated and the tubes were incubated for $8 \mathrm{~h}$. After the incubation time, slides were processed for CLSM imaging. Briefly, the slides were washed twice with $1 \mathrm{X}$ PBS to remove planktonic cells followed by staining of attached biofilm with BacLight LIVE/DEAD stain kit (L-7012; Molecular Probes) according to the manufacturer's instructions. Stained slides were imaged using Carl Zeiss confocal microscope with 63X oil immersion objective.

\section{Statistical Analysis}

Graph pad prism software (version 6.01) was used for statistical analysis. One way ANOVA and multiple comparisons were carried out. The minimum level of significance was set at $P \leq$ 0.05 . All the assays were conducted in triplicates and the results were expressed as mean $\pm \mathrm{SD}$.

\section{RESULTS}

\section{Docking Studies, Lead Optimization, and Toxicity Analysis}

In the present study, docking analysis revealed several hits that favorably occupy the active site of ComA. Each ligand was further evaluated and the compounds were modified to generate the next generation of molecules with improved binding affinity and less toxicity with the active site. For better activity of ComA inhibitors, ligands should optimally interact with at least one of the four crucial amino acid residues Q11, C17, H96, D112. Lipinski's rule of five predicts a high probability of success or failure of drug likeness for various selected molecules. Eighty one drug like ligands were subsequently guided by Lipinski's rule of three and five, pharmacokinetics (ADME) and pharmacodynamics. Rule of five states that the drug likeness for molecules to comply with three or more of the following rules: Molecular mass $<500$ dalton, high lipophilicity, $<5$ hydrogen bond donors, and $<10$ hydrogen bond acceptors.

Selected compounds were further analyzed using the QuikProp module in Schrodinger for toxicity analysis (ADMET). The properties considered for toxicity prediction were QPlogHERG, QPlogBB (Blood-brain barrier), QPlogKhsa (Human serum albumin), and human oral absorption. Among the selected 81 ligands, 7 ligands satisfied the Lipinski's rule of three and five, and the parameters considered under ADMET properties as given in Table 1. Further, ligand number 5 (ComAI, ComA Inhibitor) was selected based on the ease of synthesis with the available resources.

The selected ComAI was further used to generate derivatives with improved binding affinity by substituting various functional groups namely ComAI ${ }^{1}, \mathrm{ComAI}^{2}, \mathrm{ComAI}^{3}$, and $\mathrm{ComAI}^{4}$. All the derivatives showed better binding affinity to the active site and improved Glide score as compared to the selected ComAI and no toxicity pattern was observed for the derivatives of ComAI as given in Table 2. The ComAI as well as the derived ligands of ComAI interacts and inhibits ComA protein at Q11 through hydrogen bonding along with various other interactions in protein cleft as given in Figures 2A,B, 3A-D. Apart from the selected derivatives, one derivative (ComAI') with no interaction to the active site was also synthesized to compare and validate the computational data with in vitro data. Evidently, computational studies using docking tools exhibited excellent outcomes which shows that the selected ligands have potential binding affinity to ComA.

\section{Synthesis of 1,3-Disubstituted Ureas}

The spectral data $\left({ }^{1} \mathrm{H}-\mathrm{NMR}\right.$ and $\left.{ }^{13} \mathrm{C}-\mathrm{NMR}\right)$ of all the synthesized compounds were in full agreement with the proposed structures (Table 3). The NMR data is given in Supplementary Material.

\section{Anti-Biofilm Effects of 1, 3-Disubstitutedureas on S. mutans Biofilm Development in Static Biofilm Model}

Under static conditions, all the compounds except ComAI' were found to have antibiofilm effect. Notably, ComAI and ComAI ${ }^{1}$ showed maximum biofilm inhibition of $50-70 \%$ in all the strains with concentrations ranging from 0.23 to $3.75 \mu \mathrm{M}$ and 0.93 to $1.87 \mu \mathrm{M}$ respectively. The results were summarized in Table 4. In contrast, no biofilm inhibition was found in 5SM strain by any of the synthesized compounds. In case of eradication assay, eradication of biofilm was not found by any of the compound. Considering better biofilm inhibiting activity of ComAI and ComAI ${ }^{1}$, they were titled as best amongst all the synthesized compounds.

\section{Drug Interactions from Synergistic Studies}

The results of the checkerboard analysis were interpreted using the nonparametric methods based on both the Loewe Additivity (LA) theory and Bliss Independence (BI) theory. In the checkerboard microtiter plate assay, strong synergism was concluded in all the isolates of $S$. mutans analyzed by both the models i.e., FICI and $\Delta \mathrm{E}$, and the two models were found to be in correlation for all the tested compounds in the study.

\section{FICl}

The interpretation of the FICI was based on LA theory according to which, a FICI value of $\leq 0.5$ revealed synergy, a value of $1-4$ revealed indifference, and a value of $>4$ represented antagonism. ComAI and its derivatives showed synergistic as well as additive effects when used in combination with fluoride as shown in Table 5. In contrast, no synergism was concluded when ComAI and its derivatives were used along with chlorhexidine. Notably, when disubstituted ureas as well as fluoride were used alone, very less or no biofilm inhibition was observed in 5SM strain but considerable biofilm inhibition was observed when used in combination. 1,3-disubstituted ureas were found to inhibit the 
TABLE 1 | Final selected ligands after TOPKAT and ADMET properties as ComA inhibitors.

\begin{tabular}{|c|c|c|c|c|}
\hline Ligand No. & Structure & Glide Score & Molecular weight & Binding site and other characteristic properties \\
\hline 3 & & -3.433 & 307.34 & $\begin{array}{l}\text { H96, Q95, R93 } \\
\text { clogP: } 2.220 \\
\text { H-Donors: } 2 \\
\text { H-Acceptors: } 4 \\
\text { Rotatable bonds: } 5\end{array}$ \\
\hline 4 & & -3.532 & 318.25 & $\begin{array}{l}\text { H96, R93, L94 } \\
\text { clogP: } 2.580 \\
\text { H-Donors: } 1 \\
\text { H-Acceptors: } 6 \\
\text { Rotatable bonds: } 8\end{array}$ \\
\hline 5 & & -3.088 & 240.30 & $\begin{array}{l}\text { Q11 } \\
\text { clogP: } 3.350 \\
\text { H-Donors: } 2 \\
\text { H-Acceptors: } 3 \\
\text { Rotatable bonds: } 2\end{array}$ \\
\hline 7 & & -3.579 & 322.75 & $\begin{array}{l}\text { H96, Q95, R93 } \\
\text { clogP: } 2.860 \\
\text { H-Donors: } 1 \\
\text { H-Acceptors: } 6 \\
\text { Rotatable bonds: } 3\end{array}$ \\
\hline
\end{tabular}

biofilm in combination at a very low concentration of fluoride i.e., $62.5 \mathrm{ppm}$. In other strains used in the study, when fluoride was used alone, it showed antibacterial activity at $500 \mathrm{ppm}$ and higher concentrations, but there was no antibiofilm activity at any of the concentrations of fluoride used in the experiment. However, in synergy experiments with 1,3-disubstituted ureas, fluoride showed enhanced biofilm inhibitory effect at concentrations ranging from 31.25 to $62.5 \mathrm{ppm}$.

\section{Bliss Independence Model ( $\Delta \mathrm{E}$ Model)}

The results for BI model were summarized in Table 6. The $\Delta \mathrm{E}$ value of each tested concentration was the average of triplicate results. To summarize the interaction surface, the sums of the percentages of all statistically significant synergistic ( $\left.\sum S Y N\right)$ and antagonistic ( $\sum \mathrm{ANT}$ ) interactions were calculated. Interactions with $<100 \%$ statistically significant interactions were considered as weak, interactions with $100-200 \%$ statistically significant 
TABLE 2 | Schematic representation of ADMET and TOPKAT properties of ComAl and its derivatives.

\begin{tabular}{|c|c|c|c|c|c|c|c|}
\hline \multirow[t]{2}{*}{ Name } & \multirow[t]{2}{*}{ Structure } & \multirow{2}{*}{$\begin{array}{l}\text { Docking score/ } \\
\text { interaction site }\end{array}$} & \multicolumn{5}{|c|}{ Toxicity prediction(ADMET and TOPKAT) } \\
\hline & & & $\begin{array}{l}\text { Rule of } 3 \text { and } \\
\text { rule of } 5\end{array}$ & QPlogHERG & QPlogBB & QPlogKhsa & $\begin{array}{r}\text { Human oral } \\
\text { absorption (\% }\end{array}$ \\
\hline ComAl & & $\begin{array}{l}-3.088 \\
\text { Q11 }\end{array}$ & & & & & 100 \\
\hline
\end{tabular}

Derivatives of ComAl

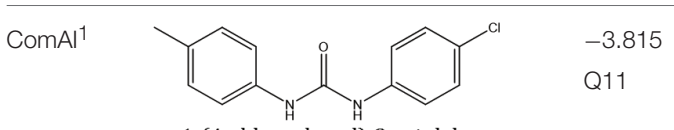

1-(4-chlorophenyl)-3-p-tolylurea

$-3.334$

Q11,Q95

ComAl $^{2}$

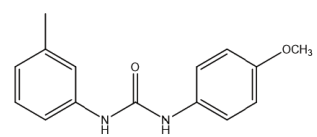

1-(4-methoxyphenyl)-3-m-tolylurea

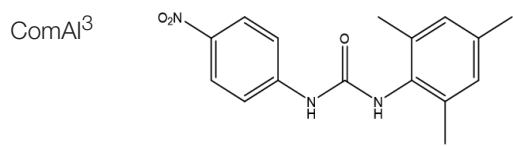

1-mesityl-3-(4-nitrophenyl)urea

\section{$-3.214$ \\ Q11, Q47, \\ L94}

ComAl $^{4}$

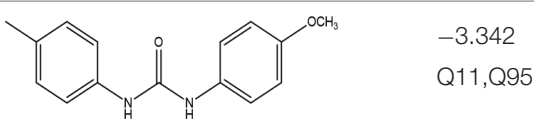

Q11,Q95
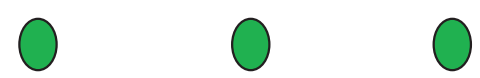

1-(4-methoxyphenyl)-3-p-tolylurea

ComAl'

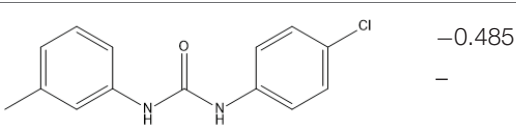

1-(4-chlorophenyl)-3-m-tolylurea

OToxicity free behavior

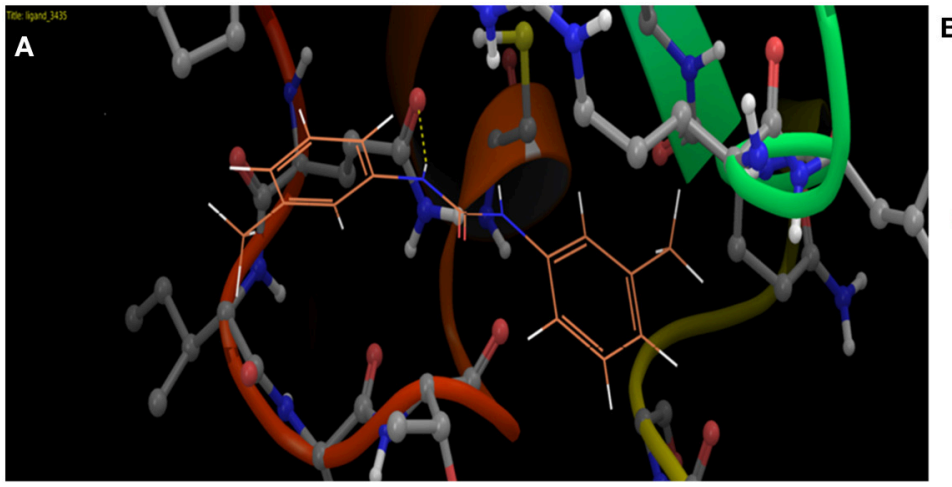

B

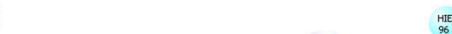

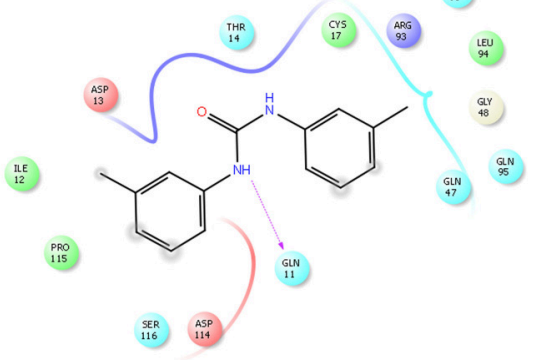

FIGURE 2 | (A) Binding of ComAl with PEP domain of ComA through Hydrogen bonding (yellow dotted line). (B) Schematic 2D representation of Ligand interaction pattern of ComAl (ComA Inhibitor) with PEP domain of ComA. 
TABLE 3 | Structures of reactants and their corresponding products.

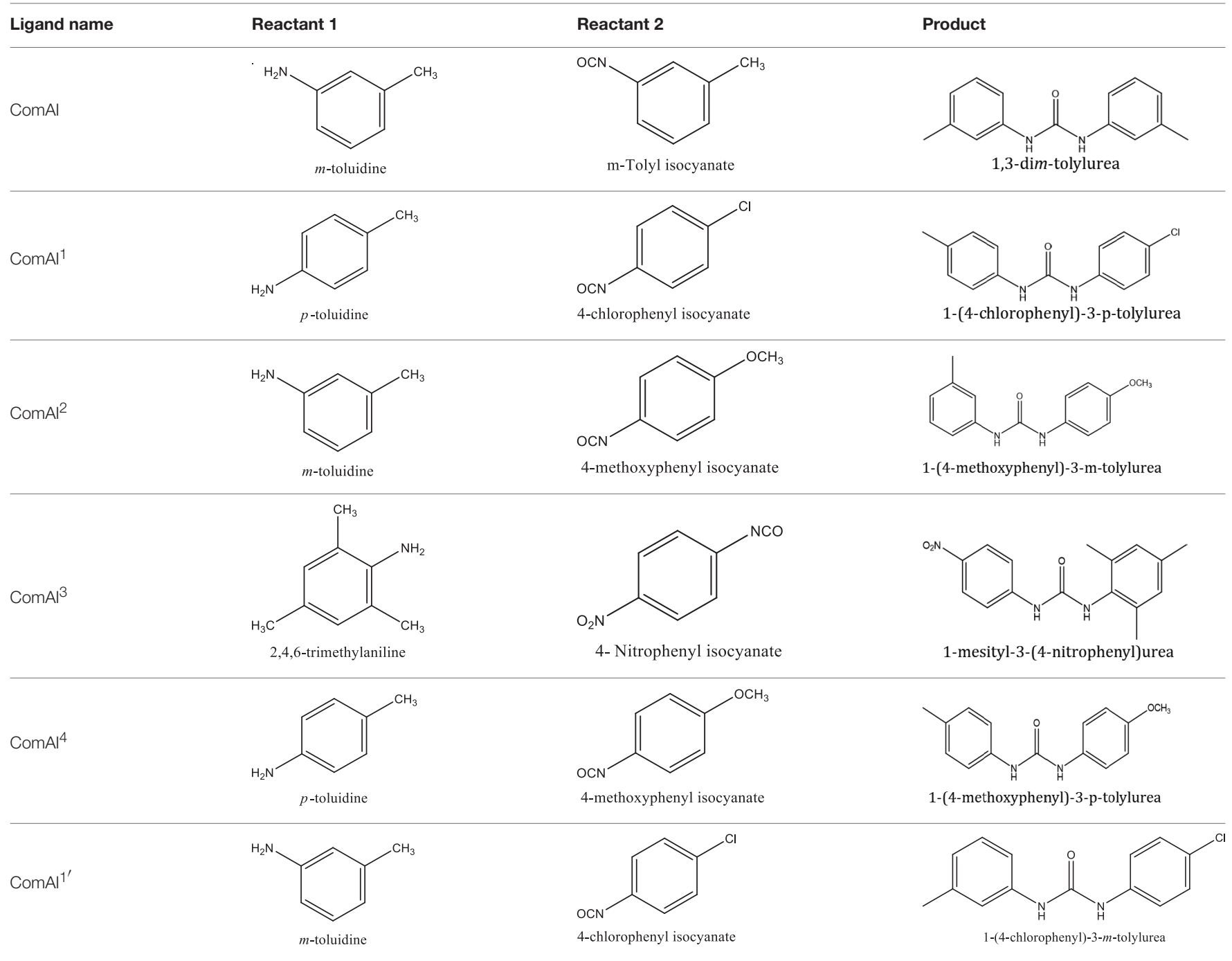

TABLE 4 | IC 50 and $\mathrm{EC}_{50}$ values of ComAl and its derivatives.

\begin{tabular}{|c|c|c|c|c|c|c|c|c|c|c|c|c|c|c|c|c|}
\hline \multirow[t]{3}{*}{ Compound } & \multicolumn{4}{|c|}{ MTCC497 } & \multicolumn{4}{|c|}{ WT } & \multicolumn{4}{|c|}{$4 \mathrm{SM}$} & \multicolumn{4}{|c|}{$5 S M$} \\
\hline & \multicolumn{2}{|c|}{$\mathrm{IC}(\mu \mathrm{M})$} & \multicolumn{2}{|c|}{$E C(\mu M)$} & \multicolumn{2}{|c|}{$\mathrm{IC}(\mu \mathrm{M})$} & \multicolumn{2}{|c|}{$\mathrm{EC}(\mu \mathrm{M})$} & \multicolumn{2}{|c|}{$I C(\mu M)$} & \multicolumn{2}{|c|}{$\mathrm{EC}(\mu \mathrm{M})$} & \multicolumn{2}{|c|}{$\operatorname{IC}(\mu \mathrm{M})$} & \multicolumn{2}{|c|}{$\mathrm{EC}(\mu \mathrm{M})$} \\
\hline & 50 & 90 & 50 & 90 & 50 & 90 & 50 & 90 & 50 & 90 & 50 & 90 & 50 & 90 & 50 & 90 \\
\hline ComAl & 3.75 & 13.5 & - & - & 14.9 & - & - & - & 0.39 & 7.5 & - & - & - & 14.8 & - & - \\
\hline ComAl $^{1}$ & 1.89 & 12.2 & - & - & - & - & - & - & 0.89 & - & - & - & - & 12.4 & - & - \\
\hline ComAl $^{2}$ & - & 13 & - & - & 11.5 & - & - & - & 1.78 & - & - & - & - & 15 & - & - \\
\hline ComAl ${ }^{4}$ & - & - & - & - & 0.46 & 14 & - & - & 0.41 & 14.5 & - & - & - & 14.5 & - & - \\
\hline ComAl ${ }^{1^{\prime}}$ & - & - & - & - & 14.7 & - & - & - & - & 13.5 & - & - & - & 11.5 & - & - \\
\hline
\end{tabular}

IC, inhibitory concentration; EC, Eradication Concentration.

Strains used: MTCC 497, standard reference strain obtained from MTCC, IMTECH, Chandigarh, India; WT, A wild type strain obtained from kindly donated by Prof. L. Jeannine Brady, University of Florida, USA.; 4SM, Multidrug resistant clinical isolate; 5SM, Fluoride resistant clinical isolate. 


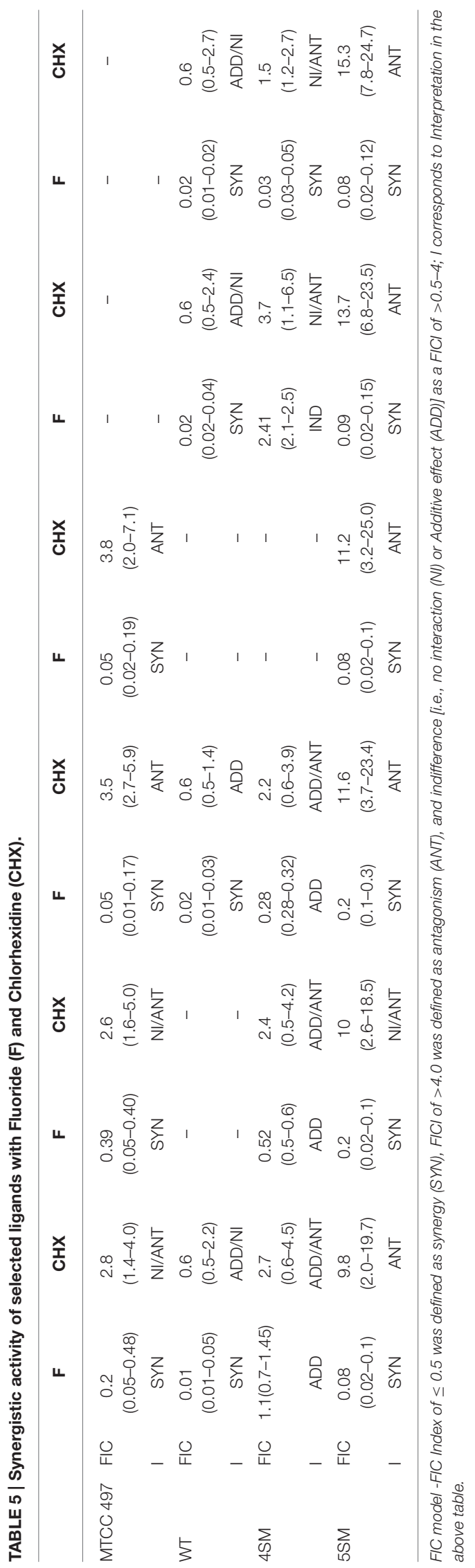

interactions were considered as moderate, and interactions with $>200 \%$ statistically significant interactions were considered as strong, as described previously. In addition, numbers of statistically significant synergistic and antagonistic combinations among all tested drug combinations were calculated for each strain. Bliss independence model was found in coherence with FIC index and confirmed strong synergism among the1,3disubstituted ureas and fluoride whereas, antagonism as well as indifference was observed in case of chlorhexidine.

\section{Confocal Laser Scanning Microscopy Imaging of Biofilms}

The confocal microscopic images of biofilm stained with BacLight Live/Dead stain were shown in Figures 4, 5. Absence of red fluorescence in the merged images from the two filter sets i.e., for live cells (Syto 9, green) and dead cells (Propidium iodide, red) showed that the treatments did not affect the survival of bacteria and the compounds are not antibacterial. This was further confirmed by plating the planktonic cells and growth inhibition was not observed (data not shown). Fifty percent biofilm inhibition was observed in ComAI $(1.89 \mu \mathrm{M})$ treated biofilms. Similar results were observed in $\operatorname{ComAI}^{1}(3.75 \mu \mathrm{M})$ treated biofilms. No significant biofilm inhibition was observed in biofilms when treated with fluoride alone whereas in case of combinatorial treatments, highly significant biofilm inhibition was observed for both S. mutans strains taken in this study.

\section{DISCUSSION}

The exploration for novel therapeutic compounds that act as antibiofilm and anti-virulent compounds such as 7-fluoroindole, 5-fluorouracil (Attila et al., 2009; Lee et al., 2012) rather than antibacterial in nature, is one amongst the major research focus these days to overcome drug resistance. Furthermore, such anti-virulent compounds when used in combination with commercially existing protective agents, results in significant reduction in the over use of these agents. In the present study, disubstitutedurea compounds were screened and synthesized and tested for its antibiofilm potential toward S. mutans. In addition, combinatorial treatment with commercially used anticaries agents such as fluoride and chlorhexidine was evaluated in vitro (Somani et al., 2010; Tong et al., 2011).

In light of our present in silico studies, we have found that disubstituted ureas have better binding affinity to ComA, a bacteriocin associated $\mathrm{ABC}$ transporter. Moreover, all the derived compounds have been shown to bind at the active site of PEP domain of ComA. Blocking of the active site will render the cleaving property of PEP domain inactivated. Hence, ComA inhibitors and its derivatives were chemically synthesized and shown to inhibit the formation of biofilm. Earlier reports by Mustafa et al. have shown anti-cancerous and enzyme inhibitory effects of 1,3-disubstituted ureas (Mustafa et al., 2014). Similarly, fluoride at higher concentrations has been reported to have antibacterial effects but has not been shown to address biofilm inhibition, a major virulence trait of the disease effectively (Zheng et al., 2015). Earlier report by Murata et al. has shown 
A

C

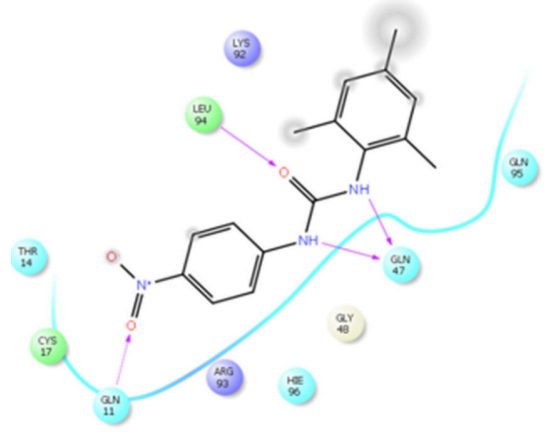

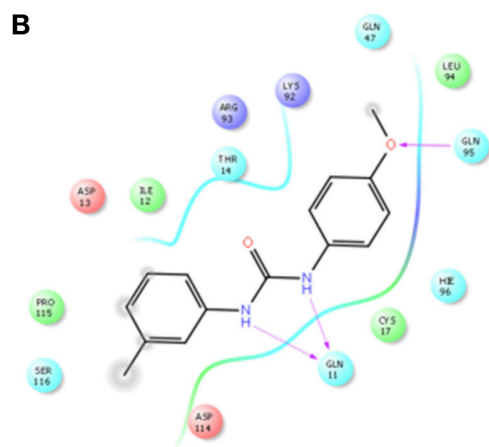

B

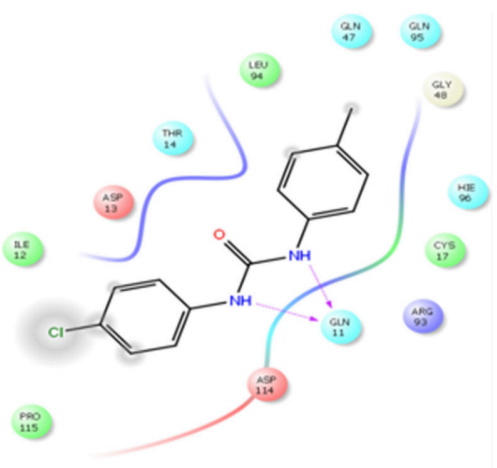

D

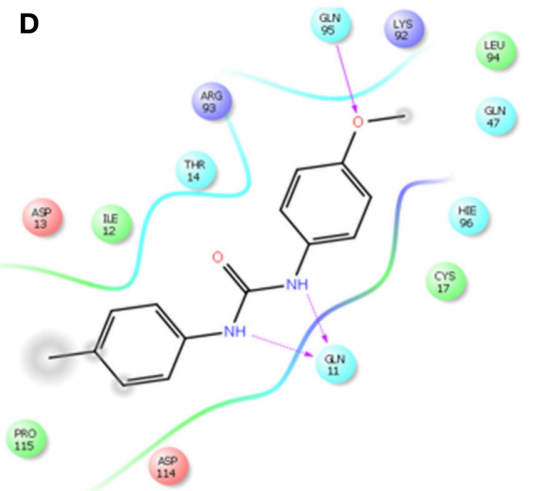

FIGURE 3 | Schematic 2D representation of interaction of derivatives of ComAl with PEP domain of ComA. (A) ComAl ${ }^{1}$; (B) ComAl${ }^{2}$; (C) $\mathrm{ComAl}^{3}$; (D) ComAl ${ }^{4}$.

TABLE 6 | Delta E model of selected compounds with Fluoride (F) and Chlorhexidine (CHX).

\begin{tabular}{|c|c|c|c|c|c|c|c|c|c|c|c|c|c|}
\hline \multirow[t]{2}{*}{ Strains } & & \multicolumn{12}{|c|}{ Nonparametric Method } \\
\hline & & $\mathbf{F}$ & $\mathrm{CHX}$ & $\mathbf{F}$ & $\mathrm{CHX}$ & $\mathbf{F}$ & $\mathrm{CHX}$ & $\mathbf{F}$ & $\mathrm{CHX}$ & $\mathbf{F}$ & $\mathrm{CHX}$ & $\mathbf{F}$ & $\mathrm{CHX}$ \\
\hline \multirow[t]{2}{*}{ MTCC 497} & $\Delta \mathrm{E}$ & 1426 & -393 & 1736 & -114 & 1771 & -531 & 1218 & -307 & - & - & - & - \\
\hline & 1 & SS & SA & SS & MA & SS & SA & SS & SA & - & & - & \\
\hline \multirow[t]{2}{*}{$4 S M$} & $\Delta \mathrm{E}$ & 98 & -329 & 55 & -521 & 78 & -283 & - & - & 87 & -489 & 1861 & -462 \\
\hline & 1 & WS & SA & WS & SA & WS & SA & - & - & WS & SA & SS & SA \\
\hline \multirow[t]{2}{*}{$5 S M$} & $\Delta \mathrm{E}$ & 1548 & -378 & 1120 & -241 & 1004 & -283 & 1217 & -228 & 1725 & -532 & 1939 & 625 \\
\hline & I & SS & SA & SS & SA & SS & SA & SS & SA & SS & SA & SS & SA \\
\hline
\end{tabular}

SS, Strong Synergism; SA, Strong Antagonism; WS, Weak Synergism; MA, Moderate Antagonism.

that fluoride at a concentration of $125 \mathrm{ppm}$ act synergistically along with 7-epiclusianone (a naturally occurring compound) to enhance the cariostatic effect of fluoride (Murata et al., 2010). Similarly, biofilm inhibition using calcium fluoride nanoparticles was observed by Kulshrestha et al. (2016).

In the present study, we have reported the effective application of chemically synthesized 1,3-disubstituted ureas that act as antibiofilm agents against standard and clinical isolates but do not have any antibacterial property which is a desirable characteristic as far as multi-drug resistance is concerned. This can be attributed to the fact that, since, these compounds were designed to selectively block the ComA, and thus, the process of cell to cell communication is inhibited leading to expression of virulence factor such as biofilm formation. Moreover, 1,3-disubstituted ureas, when used in combination with fluoride, significantly reduced the concentration of fluoride required for prevention of dental caries (1000 ppm used in the present formulations). In our present study, we have 

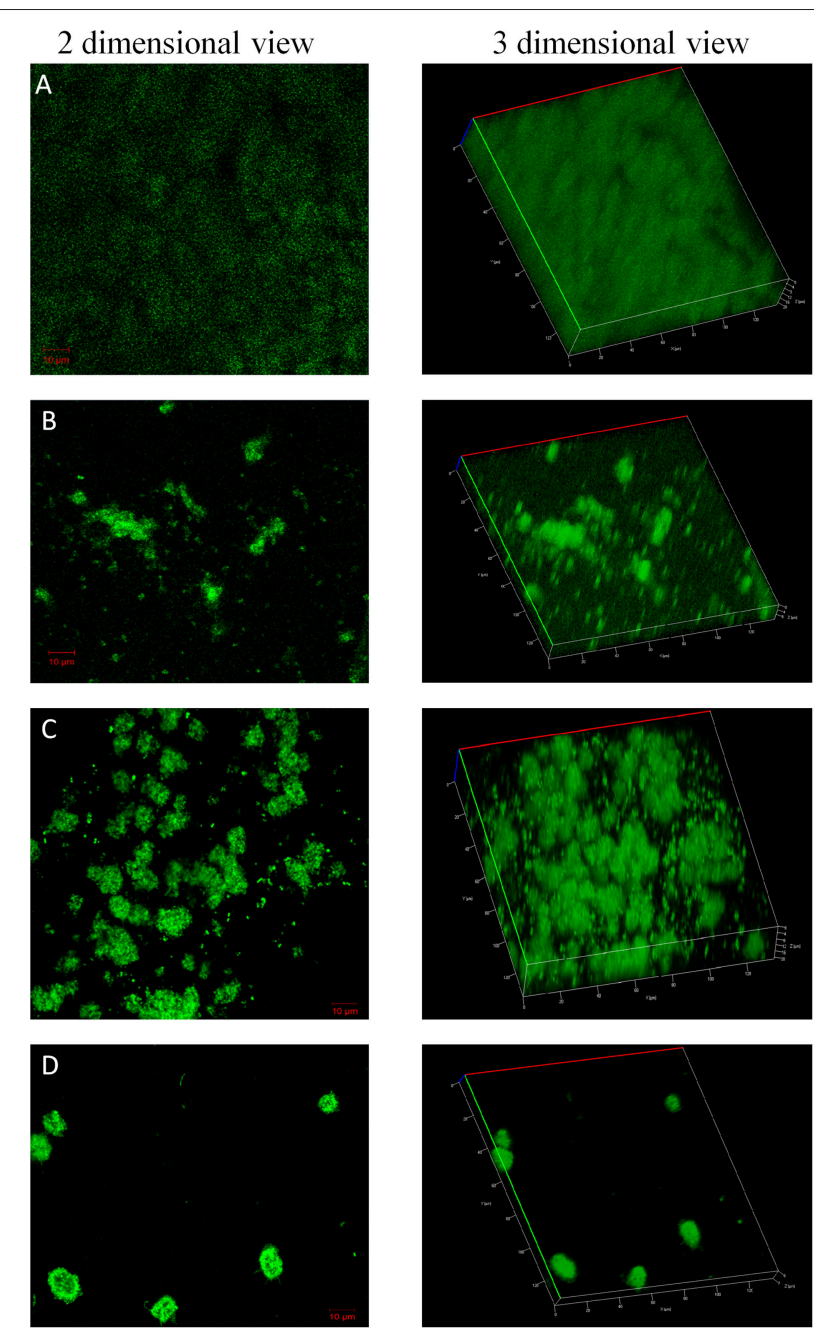

FIGURE 4 | Effect of ComAl treatments alone as well as in synergy on S. mutansMTCC 497 biofilms. Live/Dead staining of bacterial biofilm observed under confocal laser scanning microscopy (green color-Syto 9). (A) Control biofilm, (B) ComAl treated biofilm $(3.75 \mu \mathrm{M})$, (C) Fluoride treated biofilm (500 ppm), (D) Synergistic activity of ComAl and fluoride (31.25 ppm fluoride and $3.75 \mu \mathrm{M}$ ComAl)

reported the concentration of $31.25 \mathrm{ppm}$ which is found to act synergistically along with ComAI and its derivatives in all the clinical as well as standard isolates which is relatively very less fluoride concentration when compared to the presently used formulations. This suggests that the combinatorial treatment was effective and ensures the usage of antimicrobial products within the limits that may reduce or eliminate the chance of resistance development. However, similar results were not observed with chlorhexidine.

Enhancement of cario-preventive effects of fluoride by integrating the natural or synthesized compounds in oral preparations affecting the virulence of cariogenic bacteria will lead to the better dental care. Fluoride along with 1,3disubstituted ureas clearly has potential to reduce the incidence of dental caries at a very low concentrations thus, avoiding the harmful effects of increased exposure of fluoride to individuals.
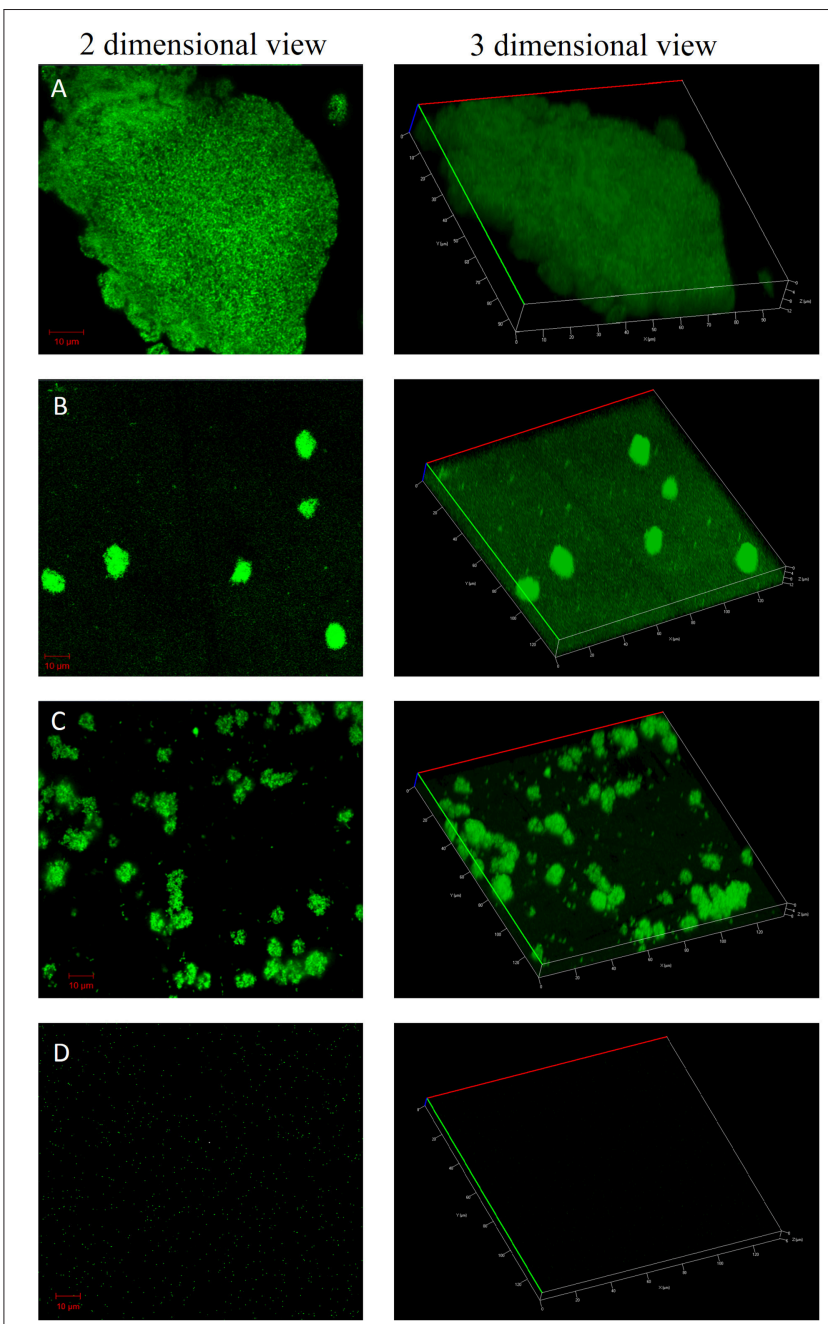

FIGURE 5 | Effect of ComAl treatments alone as well as in synergy on S. mutans 5SM (clinical isolate) biofilms. Live/Dead staining of bacterial biofilm observed under confocal laser scanning microscopy (green color-Syto 9). (A) Control biofilm, (B) ComAl treated biofilm (15 $\mu \mathrm{M})$, (C) Fluoride treated biofilm (500 ppm), (D) Synergistic activity of ComAl and fluoride (31.25 ppm fluoride and $15 \mu \mathrm{M}$ ComAl).

Collectively, based on the in vitro studies, our data shows that 1,3-disubstituted ureas reduces biofilm formation when used alone or in combination with fluoride without reducing its cariostatic properties. The combination of fluoride with 1,3disubstituted ureas may provide a potentially useful alternative to the current chemo-based anti-caries strategies to prevent dental caries disease. However, additional studies are essential to elucidate the molecular mechanisms of action of these agents and optimize the combinations. This would enhance the present effectiveness of anti-caries chemotherapy.

\section{AUTHOR CONTRIBUTIONS}

All authors listed, have made substantial, direct and intellectual contribution to the work, and approved it for publication. 


\section{ACKNOWLEDGMENTS}

Author, GK is a recipient of the University Grants Commission Maulana Azad National Fellowship (UGC-MANF) (MANF2014-15-SIK-PUN-31423) from the University Grants Commission, Government of India and the support is duly acknowledged. We sincerely thank the SASTRA University and its management for providing us the infrastructure needed to carry out our research work. We also acknowledge the DST-PURSE sponsored CLSM facility (No. SR/FT/LS-113/2009)

\section{REFERENCES}

Attila, C., Ueda, A., and Wood, T. K. (2009). 5-Fluorouracil reduces biofilm formation in Escherichia coli K-12 through global regulator AriR as an antivirulence compound. Appl. Microbiol. Biotechnol. 82, 525-533. doi: 10.1007/s00253-009-1860-8

Auty, M., Gardiner, G., McBrearty, S., O’Sullivan, E., Mulvihill, D., Collins, J., et al. (2001). Direct in situ viability assessment of bacteria in probiotic dairy products using viability staining in conjunction with confocal scanning laser microscopy. Appl. Environ. Microbiol. 67, 420-425. doi: 10.1128/AEM.67.1.420-425.2001

Bernstein, F. C., Koetzle, T. F., Williams, G. J., Meyer, E. F., Brice, M. D., Rodgers, J. R., et al. (1978). The Protein Data Bank: a computer-based archival file for macromolecular structures. Arch. Biochem. Biophys. 185, 584-591. doi: 10.1016/0003-9861(78)90204-7

Cavalli, V., Rodrigues, L. K. A., Paes-Leme, A. F., Soares, L. E. S., Martin, A. A., Berger, S. B., et al. (2011). Effects of the addition of fluoride and calcium to lowconcentrated carbamide peroxide agents on the enamel surface and subsurface. Photomed. Laser Surg. 29, 319-325. doi: 10.1089/pho.2010.2797

Chen, L., Ren, Z., Zhou, X., Zeng, J., Zou, J., and Li, Y. (2016). Inhibition of Streptococcus mutans biofilm formation, extracellular polysaccharide production, and virulence by an oxazole derivative. Appl. Microbiol. Biotechnol. 100, 857-867. doi: 10.1007/s00253-015-7092-1

Friesner, R. A., Banks, J. L., Murphy, R. B., Halgren, T. A., Klicic, J. J., Mainz, D. T., et al. (2004). Glide: a new approach for rapid, accurate docking and scoring. 1. Method and assessment of docking accuracy. J. Med. Chem. 47, 1739-1749. doi: $10.1021 / \mathrm{jm} 0306430$

Friesner, R. A., Murphy, R. B., Repasky, M. P., Frye, L. L., Greenwood, J. R., Halgren, T. A., et al. (2006). Extra precision glide: docking and scoring incorporating a model of hydrophobic enclosure for protein-ligand complexes. J. Med. Chem. 49, 6177-6196. doi: 10.1021/jm051256o

Goldoni, M., and Johansson, C. (2007). A mathematical approach to study combined effects of toxicants in vitro: evaluation of the Bliss independence criterion and the Loewe additivity model. Toxicol. In Vitro 21, 759-769. doi: 10.1016/j.tiv.2007.03.003

Han, T. K., Zhang, C., and Dao, M. L. (2006). Identification and characterization of collagen-binding activity in Streptococcus mutans wall-associated protein: a possible implication in dental root caries and endocarditis. Biochem. Biophys. Res. Commun. 343, 787-792. doi: 10.1016/j.bbrc.2006.03.025

Hartenfeller, M., and Schneider, G. (2011). “De novo drug design," in Chemoinformatics and Computational Chemical Biology, ed J. Bajorath (Frankfurt; Zürich: Springer), 299-323.

Hasan, S., Danishuddin, M., Adil, M., Singh, K., Verma, P. K., and Khan, A. U. (2012). Efficacy of E. officinalis on the cariogenic properties of Streptococcus mutans: a novel and alternative approach to suppress quorumsensing mechanism. PLoS ONE 7:e40319. doi: 10.1371/journal.pone.00 40319

Irwin, J. J., and Shoichet, B. K. (2005). ZINC-a free database of commercially available compounds for virtual screening. J. Chem. Inf. Model. 45, 177-182. doi: $10.1021 / \mathrm{ci049714+}$

Ishii, S., Yano, T., Ebihara, A., Okamoto, A., Manzoku, M., and Hayashi, H. (2010). Crystal structure of the peptidase domain of Streptococcus ComA, a bifunctional ATP-binding cassette transporter involved in the quorumsensing pathway. J. Biol. Chem. 285, 10777-10785. doi: 10.1074/jbc.M109. 093781 at Bharathidasan University, Trichy, Tamil Nadu for confocal imaging.

\section{SUPPLEMENTARY MATERIAL}

The Supplementary Material for this article can be found online at: http://journal.frontiersin.org/article/10.3389/fmicb. 2016.00861

The supplementary information includes the NMR data $(1 \mathrm{H}$ and 13C NMR) for all the synthesized compounds.

Jennings, M. C., Ator, L. E., Paniak, T. J., Minbiole, K. P., and Wuest, W. M. (2014). Biofilm-eradicating properties of quaternary ammonium amphiphiles: simple mimics of antimicrobial peptides. Chembiochem 15, 2211-2215. doi: 10.1002/cbic. 201402254

Kaur, G., Rajesh, S., and Princy, S. A. (2015). Plausible drug targets in the Streptococcus mutans quorum sensing pathways to combat dental biofilms and associated risks. Indian J. Microbiol. 55, 349-356. doi: 10.1007/s12088-0150534-8

Kleerebezem, M., Quadri, L. E., Kuipers, O. P., and De Vos, W. M. (1997). Quorum sensing by peptide pheromones and two-component signaltransduction systems in Gram-positive bacteria. Mol. Microbiol. 24, 895-904. doi: 10.1046/j.1365-2958.1997.4251782.x

Kolodkin-Gal, I., Cao, S., Chai, L., Böttcher, T., Kolter, R., Clardy, J., et al. (2012). A self-produced trigger for biofilm disassembly that targets exopolysaccharide. Cell 149, 684-692. doi: 10.1016/j.cell.2012.02.055

Kotake, Y., Ishii, S., Yano, T., Katsuoka, Y., and Hayashi, H. (2008). Substrate recognition mechanism of the peptidase domain of the quorum-sensingsignal-producing ABC transporter ComA from Streptococcus. Biochemistry 47, 2531-2538. doi: 10.1021/bi702253n

Kulshrestha, S., Khan, S., Hasan, S., Khan, M. E., Misba, L., and Khan, A. U. (2016). Calcium fluoride nanoparticles induced suppression of Streptococcus mutans biofilm: an in vitro and in vivo approach. Appl. Microbiol. Biotechnol. 100, 1901-1914. doi: 10.1007/s00253-015-7154-4

Lee, J.-H., Kim, Y.-G., Cho, M. H., Kim, J.-A., and Lee, J. (2012). 7-fluoroindole as an antivirulence compound against Pseudomonas aeruginosa. FEMS Microbiol. Lett. 329, 36-44. doi: 10.1111/j.1574-6968.2012.02500.x

Lynch, D. J., Michalek, S. M., Zhu, M., Drake, D., Qian, F., and Banas, J. A. (2013). Cariogenicity of Streptococcus mutans glucan-binding protein deletion mutants. Oral Health Dent. Manag. 12, 191-199.

Miller, M. B., and Bassler, B. L. (2001). Quorum sensing in bacteria. Annu. Rev. Microbiol. 55, 165-199. doi: 10.1146/annurev.micro.55.1.165

Mitsuhata, C., Puteri, M. M., Ohara, Y., Tatsukawa, N., and Kozai, K. (2014). Possible involvement of enolase in fluoride resistance in Streptococcus mutans. Pediatr. Dent. J. 24, 12-16. doi: 10.1016/j.pdj.2013.10.002

Murata, R. M., Branco-de-Almeida, L. S., Franco, E. M., Yatsuda, R., dos Santos, M. H., de Alencar, S. M., et al. (2010). Inhibition of Streptococcus mutans biofilm accumulation and development of dental caries in vivo by 7 -epiclusianone and fluoride. Biofouling 26, 865-872. doi: 10.1080/08927014.2010.527435

Mustafa, S., Perveen, S., and Khan, A. (2014). Synthesis, enzyme inhibition and anticancer investigation of unsymmetrical 1, 3-disubstituted ureas. J. Serb. Chem. Soc. 79, 1-10. doi: 10.2298/JSC121212076M

Osguthorpe, D. J., Sherman, W., and Hagler, A. T. (2012). Exploring protein flexibility: incorporating structural ensembles from crystal structures and simulation into virtual screening protocols. J. Phys. Chem. B 116, 6952-6959. doi: $10.1021 /$ jp3003992

Persson, G. R. (2012). Rheumatoid arthritis and periodontitis - inflammatory and infectious connections. Review of the literature. J. Oral Microbiol. 4. doi: 10.3402/jom.v4i0.11829

Qi, F., Kreth, J., Lévesque, C. M., Kay, O., Mair, R. W., Shi, W., et al. (2005). Peptide pheromone induced cell death of Streptococcus mutans. FEMS Microbiol. Lett. 251, 321-326. doi: 10.1016/j.femsle.2005.08.018

Randall, J., Seow, W., and Walsh, L. (2014). Antibacterial activity of fluoride compounds and herbal toothpastes on Streptococcus mutans: an in vitro study. Aust. Dent. J. 60, 368-374. doi: 10.1111/adj.12247 
Rasmussen, T. B., and Givskov, M. (2006). Quorum sensing inhibitors: a bargain of effects. Microbiology 152, 895-904. doi: 10.1099/mic.0.28601-0

Ravichandiran, V., Shanmugam, K., and Princy Solomon, A. (2013). Screening of SdiA inhibitors from melia dubia seeds extracts towards the hold back of uropathogenic E. coli quorum sensing-regulated factors. Med. Chem. 9, 819-827. doi: 10.2174/1573406411309060006

Santos, A., Oliveira, B., and Nadanovsky, P. (2013). Effects of low and standard fluoride toothpastes on caries and fluorosis: systematic review and metaanalysis. Caries Res. 47, 382-390. doi: 10.1159/000348492

Senadheera, D., and Cvitkovitch, D. G. (2008). Quorum sensing and biofilm formation by Streptococcus mutans. Adv. Exp. Med. Biol. 631, 178-188. doi: 10.1007/978-0-387-78885-2_12

Singh, S. P., and Konwar, B. K. (2012). Molecular docking studies of quercetin and its analogues against human inducible nitric oxide synthase. Springerplus 1, 1-10. doi: 10.1186/2193-1801-1-69

Somani, R., Garewal, R. S., Jaidka, S., and Pasricha, J. (2010). Comparative evaluation of aqueous extract of terminalia chebula and chlorhexidine oral rinse as an anti caries agent-an in vivo research study. J. Res. Educ. Indian Med. (Estt. 1982) $16,73-78$.

Subramaniam, S., Keerthiraja, M., and Sivasubramanian, A. (2014), Synergistic antibacterial action of $\beta$-sitosterol-D-glucopyranoside isolated from Desmostachya bipinnata leaves with antibiotics against common human pathogens. Revista Brasileira Farmacognosia 24, 44-50. doi: 10.1590/0102-695X20142413348

Sun, S., Li, Y., Guo, Q., Shi, C., Yu, J., and Ma, L. (2008). In vitro interactions between tacrolimus and azoles against Candida albicans determined by different methods. Antimicrob. Agents Chemother. 52, 409-417. doi: 10.1128/AAC.01070-07
Tong, Z., Zhou, L., Jiang, W., Kuang, R., Li, J., Tao, R., et al. (2011). An in vitro synergetic evaluation of the use of nisin and sodium fluoride or chlorhexidine against Streptococcus mutans. Peptides 32, 2021-2026. doi: 10.1016/j.peptides.2011.09.002

Wang, H., Jørgensen, C., Ciofu, O., Christophersen, L., Kolpen, M., Jensen, P., et al. (2015). WS02. 5 Pharmacodynamics of ceftazidime combined with $\beta$-lactamase inhibitors in biofilm Pseudomonas aeruginosa in vitro. J. Cystic Fibrosis 14:S4. doi: 10.1016/S1569-1993(15)30011-4

Xiao, L., Nickbarg, E., Wang, W., Thomas, A., Ziebell, M., Prosise, W. W., et al. (2011). Evaluation of in vitro PXR-based assays and in silico modeling approaches for understanding the binding of a structurally diverse set of drugs to PXR. Biochem. Pharmacol. 81, 669-679. doi: 10.1016/j.bcp.2010. 12.003

Zheng, X., Cheng, X., Wang, L., Qiu, W., Wang, S., Zhou, Y., et al. (2015). Combinatorial effects of arginine and fluoride on oral bacteria. J. Dent. Res. 94, 344-353. doi: 10.1177/0022034514561259

Conflict of Interest Statement: The authors declare that the research was conducted in the absence of any commercial or financial relationships that could be construed as a potential conflict of interest.

Copyright (C) 2016 Kaur, Balamurugan, Uma Maheswari, Anitha and Princy. This is an open-access article distributed under the terms of the Creative Commons Attribution License (CC BY). The use, distribution or reproduction in other forums is permitted, provided the original author(s) or licensor are credited and that the original publication in this journal is cited, in accordance with accepted academic practice. No use, distribution or reproduction is permitted which does not comply with these terms. 